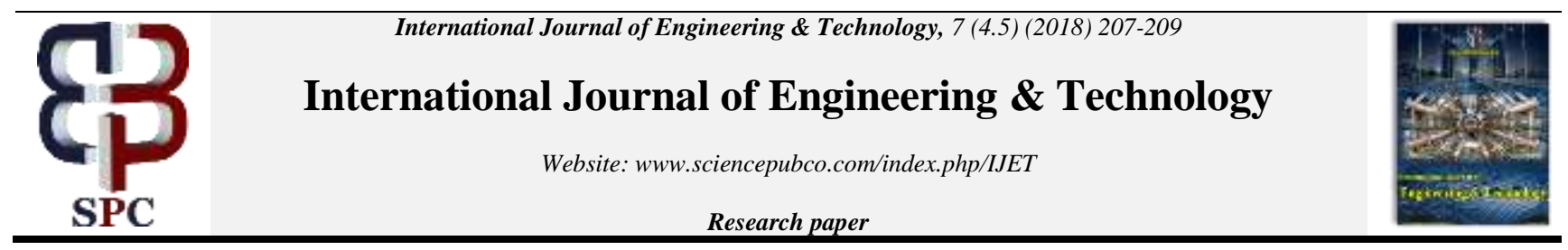

\title{
Energy Efficient Design of Wireless Sensor Network: Clustering
}

\author{
Ram Murthy Garimella ${ }^{1 *}$, Damodar Reddy Edla ${ }^{2}$, Venkatanareshbabu Kuppili ${ }^{3}$ \\ ${ }^{1}$ International Institute of Information Technology, Gachibowli, Hyderbad - 500 032, India \\ ${ }^{2,3}$ National Institute of Technology Goa, Farmagudi, Ponda, Goa-403401 \\ *Corresponding author E-mail:rammurthy@iiit.ac.in
}

\begin{abstract}
One of the significant criteria of wireless sensor network is energy efficiency. Focused on the energy problem, researchers design a few algorithms based on clustering in the present era but lack of proper mathematically rigor justification. To fill this research gap, a system and method has been proposed with a mathematical proof induced from the concept of Hessian matrix of multi variable calculus for Energy efficient techniques of data gathering or routing in Wireless Sensor Networks. It is assumed that Wireless Sensor Nodes are distributed in a multidimensional space. Illustrations verify that the proposed system mathematical design for any clustering approach promises a low-energy communication structure.
\end{abstract}

Keywords: Clustering; Sensor; Gateway; Hessian Matrix

\section{Introduction}

Wireless Sensor Networks (WSNs) are distributed network of various sensors which have the ability to sense, process and connect with other sensors. They are widely used in field of including engineering, health and environment. WSNs have the ability to communicate or monitor remotely located sensors with comparatively reasonable cost. Sensors or nodes in a WSN is basically involved in accomplishing four main tasks namely, data collection, data sending, data receiving and processing of data. In order to fulfil these tasks, sensors or nodes must proficiently exploit the available resources like memory, processor, energy etc. Efficient use of resource will in turn increase the lifetime and production strength of sensors or nodes. Over the past the main issue of maintain sensors useful is to maximize productivity whilst minimizing consumption of energy. Researchers have developed various algorithms on various layers of WSN in order to utilize energy resourcefully. Some of the energy-constrained sensors are developed where sensors are expected to run autonomously for long periods. In order to overcome energy issue WSNs are designed for specific applications ranging from small-size healthcare surveillance systems to large-scale environmental monitoring. Thus, for a particular application WSN has to satisfy a set of requirements that differs from one application to another.

In wireless sensor network (WSNs), having battery powered sensors are coordinated to gather information about a sensed variable and communicate the information to a base station/sink, in many applications, replacement of battery in the sensor is difficult or even not possible. Thus, the design of protocols in such network must be energy efficient. Various researchers proposed hierarchical energy efficient routing/fusion algorithms such as "Low Energy Clustering Hierarchy (LEACH)", HEED etc. In such algorithms, a group of wireless sensor nodes forming a cluster have a representative leader called cluster head. Periodically the cluster head is rotated among the members of the cluster. The cluster heads essentially participate in the data fusion and routing the sensed packets to the base station/ sink. In many interesting applications, the sensors are geographically distributed with certain distance between them. In most existing energy efficient algorithms, distance between sensors is not taken into account for deciding the cluster head. In this research paper we formulate the problem of choosing the cluster head (taking into account the distance between sensors along with other factors) as a quadratic optimization problem and solve the problem. The cluster head is chosen to be centroids of sensor position coordinates.

\section{Literature review}

Various approaches have been developed in past to optimize the energy of WSN. Anastasi et al. [1] have presented an extensive survey on WSNs and various approaches used for energy consumption, like duty cycling, data driven approaches and some of the mobility based approaches. A clustering based energy efficient algorithm [2] has been applied where WSNs are organized in a two-layer manner with clustering algorithm and then, the missing data was recovered based on two-layer structure. Wireless sensor networks use battery-operated computing and sensing devices for them to be operated in ad hoc manner. So, a "Medium-Access Control (MAC)" protocol- S-MAC has been designed for wireless sensor networks [3]. Another energy efficiency approach has been proposed [4] where analysis have been done by developing a method of controlling network lifetime and found node density as a major parameter which has significant role to control network lifetime. They proposed a "Probability Density Function (PDF)" and derived its intrinsic characteristics like covariance, mean etc. Also they have developed a node deployment algorithm based on PDF to avoid energy problem.

For maximizing the lifetime of network, consumption of energy should be optimal. For achieving the goal of optimal energy con- 
sumption various work have been done in past. One of the most common method for reducing energy consumption is load balancing. For cluster based WSNs, cluster heads perform the four main tasks of collection, aggregation, communication and processing of data with the base station and with its member nodes. Hence to maximize the lifetime of network, load balancing is performed within gateways. In order to achieve this goal, a "Shuffled Frog Leaping Algorithm (SFLA)" is enhanced by adapting the frog's population generation and off-spring generation phases in SFLA and by presenting a transfer phase [5]. To evaluate the quality of solution evaluated by proposed method, a novel fitness function has been designed. An energy efficient algorithm based on time synchronization has been proposed in order to conserve energy when nodes are inactive [6]. "Multi-Factors Backpressure Scheduling (MFBS)" algorithm have been proposed [7], where smart WSNs are designed which uses new link weights for energy scheduling among various sensors. Scheduling decisions are made by considering differential nodal residual energy as well as the shortest path between neighbors (nodes) jointly. The neighboring node having higher residual energy and minimum path length will be transferred the next packet.

A genetic algorithm inspired clustering algorithm has been given by authors in [8]. The algorithm uses genetic algorithm based approach to find optimal clusters that consumes less energy. Few authors have applied data aggregation based approaches for efficient energy WSNs. In data aggregation data from different sources are aggregated to an intermediate node. A routing algorithm called cluster-chain mobile agent routing (CCMAR) is proposed by [9]. This algorithms combines both LEACH and "Power-Efficient GAthering in Sensor Information Systems (PEGASIS)". Similarly a ring clustering based approach for energy efficient WSN has been given in [10]. As signals move in circular direction hence a ring domain communication topology can effectively use $360^{\circ}$ signal transduction while also reducing message collision during the transmission process. Most of the previous work on clustering has adopted a two-layer hierarchy, so threelayer scheme has been introduced in [11] and applied three layered architecture on hybrid of centralized gridding and distributed clustering. The grid heads are determined in a centralized manner, and then the $\mathrm{CHs}$ are determined in a distributed manner. Using threelayer hierarchy, the number of nodes that communicate with base station reduced, and results in energy conservation.

\section{Proposed approach}

Consider a WSN system in which all the sensor nodes are randomly deployed together with a couple of gateways and as soon as they are positioned, they become static. All sensor nodes are assigned to a gate way when they are within communication range. Therefore, each sensor node has a couple of gateways and it is allocated to only one gateway selected amongst them. The sensor nodes gather the significant local data and transfer it to the corresponding gate ways. On getting the data, the gateways process them to reduce the redundant data within their cluster. It is assumed that a wireless communication link is established between two nodes only if they are within the inter cluster distance (communication range). An example for clustered based WSN is presented in Fig 1.

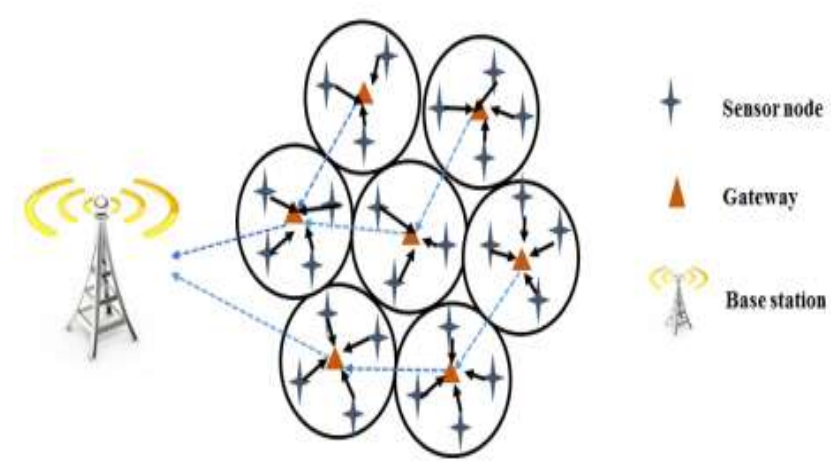

Fig 1: Cluster based WSN with gateways

\subsection{Problem formulation}

Consider $\mathrm{N}$ data points or sensor nodes in M-dimensional pattern space i.e. M-dimensional vectors that are totally $\mathrm{N}$ of them. $(\mathrm{S} 1)^{-},(\mathrm{S} 2)^{-}, \ldots .,(\mathrm{S} N \mathrm{~N})^{-}$, with $(\mathrm{S} \mathrm{j})^{-}=\left[\left(\mathrm{s}_{\mathrm{j}} 1\right)^{-},(\mathrm{s} \mathrm{j} 2)^{-}, \ldots .\right.$. $\left.(\mathrm{s} j \mathrm{M})^{-},\right]$for $1 \leq \mathrm{j} \leq \mathrm{N}$. Let $\left(\mathrm{G}_{-} 0\right)^{-}$be the desired centroid or gateway i.e. $\left(\mathrm{G}_{-} 0\right)=\left[\mathrm{g}=01, \mathrm{~g} \_02, \ldots, \mathrm{g} \_\mathrm{M}\right]$.

Theorem: Consider $\mathrm{n}$ sensor nodes positioned in a $\mathrm{M}$ Dimensional Space. Centroid of $\mathrm{N}$ points in M-dimension minimizes the sum of squared distances from the points to the centroid (Gateway) in a Euclidian distance based cluster of all the $\mathrm{n}$-sensor nodes within its communication range.

Proof: Compute $\overline{G_{0}}$, i.e. centroid in such a way that sum of squares of Euclidean distances (from centroid to patter) is minimized.

$\mathrm{J}\left(\overline{\mathrm{G}_{0}}\right)=\sum_{\mathrm{j}=1}^{\mathrm{N}} \sum_{\mathrm{i}=1}^{\mathrm{M}}\left[\mathrm{s}_{\mathrm{ji}}-\mathrm{g}_{\mathrm{oi}}\right] 2$

We could like to solve this unconstrained optimization problem. Differentiating we have

$\frac{\delta \mathrm{J}\left(\overline{\mathrm{G}_{0}}\right)}{\delta \delta \mathrm{J}\left(\mathrm{g}_{0 \mathrm{i}}\right)}=\sum_{\mathrm{j}=1}^{\mathrm{N}} 2\left[\mathrm{~s}_{\mathrm{ji}}-\mathrm{g}_{0 \mathrm{i}}\right](-1)$ for $1 \leq \mathrm{i} \leq \mathrm{M}$

Setting it to zero, we have

$\sum_{\mathrm{j}=1}^{\mathrm{N}} S_{j i}=(\mathrm{N})\left(g_{0 \mathrm{i}}\right)$ for $1 \leq \mathrm{i} \leq \mathrm{M}$

Thus we can say that

$\mathrm{g}_{0 \mathrm{i}}=\frac{1}{\mathrm{~N}} \sum_{\mathrm{j}=1}^{\mathrm{N}} \mathrm{S}_{\mathrm{ji}}$ for $1 \leq \mathrm{i} \leq \mathrm{M}$

i.e. $g_{01}=\frac{1}{N} \sum_{j=1}^{N} s_{j 1}$ and so on.

We will now prove that like centroid

$\left(\overline{G_{0}}=\left[g_{01}, g_{02}, \ldots, g_{0 M}\right]\right)$

is indeed a global minimum point i.e. we compute second partial derivative and reason that hessian matrix is positive definite i.e. eigenvalues are all positive and we have a diagonal Hessian matrix.

$$
\begin{aligned}
& \frac{\delta^{2} \mathrm{~J}\left(\overline{\mathrm{G}_{0}}\right)}{\delta \mathrm{g}_{0 \mathrm{i}}^{2}}=\sum_{\mathrm{j}=1}^{\mathrm{N}} \frac{\delta}{\delta \mathrm{G}_{0 \mathrm{i}}}\left[-2\left(\mathrm{~s}_{\mathrm{ji}}-\mathrm{g}_{0 \mathrm{i}}\right)\right]= \\
& \sum_{\mathrm{j}=1}^{\mathrm{N}}+2=2 \mathrm{~N}
\end{aligned}
$$


Also, it readily follows that

$$
\frac{\delta^{2} \mathrm{~J}\left(\overline{\mathrm{G}_{0}}\right)}{\delta \mathrm{g}_{0 \mathrm{k}} \delta \mathrm{g}_{0 \mathrm{i}}}=0
$$

For $\mathrm{k} \neq \mathrm{i}$. $\left[\begin{array}{cccc}2 \mathrm{~N} & 0 & \ldots & 0 \\ 0 & 2 \mathrm{~N} \ldots & 0 \\ 0 & 0 \ldots & 2 \mathrm{~N}\end{array}\right]$

\section{Conclusion}

In this paper, we have proposed a new approach to improve the energy efficiency of the wireless sensor network by placing the base stations in the appropriate locations such that the squared Euclidean distances from the sensors to the base stations are minimized. We have used the concept of Hessian matrix of multi variable calculus to improve the energy efficiency and identify the base station locations such that the energy consumption is minimized. The proposed methodology is supported with problem formulation and necessary proofs. The proposed mathematical design will significally support and improve the low-energy networks.

\section{References}

[1] Anastasi, Giuseppe, et al. "Energy conservation in wireless sensor networks: A survey." Ad hoc networks 7.3 (2009): 537-568.

[2] X. Liu, J. Li, Z. Dong and F. Xiong, "Joint Design of EnergyEfficient Clustering and Data Recovery for Wireless Sensor Networks," in IEEE Access, vol. 5, pp. 3646-3656, 2017.

[3] Wei Ye, J. Heidemann and D. Estrin, "An energy-efficient MAC protocol for wireless sensor networks," Proceedings.Twenty-First Annual Joint Conference of the IEEE Computer and Communications Societies, 2002, pp. 1567-1576 vol.3.

[4] S. Halder and S. DasBit, "Design of a Probability Density Function Targeting Energy-Efficient Node Deployment in Wireless Sensor Networks," in IEEE Transactions on Network and Service Management, vol. 11, no. 2, pp. 204-219, June 2014.

[5] D. R. Edla, A. Lipare, R. Cheruku and V. Kuppili, "An Efficient Load Balancing of Gateways Using Improved Shuffled Frog Leaping Algorithm and Novel Fitness Function for WSNs," in IEEE Sensors Journal, vol. 17, no. 20, pp. 6724-6733, Oct.15, 152017.

[6] T. Xia and S. He, "New energy-efficient time synchronization algorithm design for wireless sensor networks," 2017 32nd Youth Academic Annual Conference of Chinese Association of Automation (YAC), Hefei, 2017, pp. 490-495.

[7] F. Alassery, "A new link weight factor in backpressure scheduling algorithm for energy-efficient design of Smart Wireless Sensor Networks," 2016 IEEE Smart Energy Grid Engineering (SEGE), Oshawa, ON, 2016, pp. 365-370.

[8] S. B. Amsalu, W. K. Zegeye, D. Hailemariam and Y. Astatke, "Design and performance evaluation of an energy efficient routing protocol for Wireless Sensor Networks," 2016 Annual Conference on Information Science and Systems (CISS), Princeton, NJ, 2016, pp. 48-53.

[9] S. Sasirekha and S. Swamynathan, "Cluster-chain mobile agent routing algorithm for efficient data aggregation in wireless sensor network," in Journal of Communications and Networks, vol. 19, no. 4, pp. 392-401, August 2017.

[10] W. Zhang, L. Li, G. Han and L. Zhang, "E2HRC: An EnergyEfficient Heterogeneous Ring Clustering Routing Protocol for Wireless Sensor Networks," in IEEE Access, vol. 5, pp. 1702-1713, 2017.

[11] J. S. Lee and T. Y. Kao, "An Improved Three-Layer Low-Energy Adaptive Clustering Hierarchy for Wireless Sensor Networks," in IEEE Internet of Things Journal, vol. 3, no. 6, pp. 951-958, Dec. 2016 . 